\title{
Size Dependent Mechanical Properties and Photomechanical Fatigue of Diarylethene Molecular Crystals Using Atomic Force Microscopy
}

Thiranjeewa Lansakara ${ }^{1}$, Fei Tong ${ }^{2}$, Christopher Bardeen ${ }^{2}$ and Alexei Tivanski ${ }^{1}$

${ }^{1}$ The University of Iowa, Iowa City, Iowa, United States, ${ }^{2}$ University of California Riverside, Riverside, California, United States

Nano-dimensional materials due to their unique physical-chemical properties recently attracted a significant interest for applications in the fields of medicine, pharmaceutics and material science. Size reduction to nanoscale can lead to remarkably different properties compared to their bulk counterparts. Photo responsive molecular solids in nano-dimension are particularly intriguing, since such materials can undergo solid-solid chemical transformations while performing multiple types of motion (i.e. bending, curling, expansion, fragmentation etc.) leading to direct conversion of light energy to mechanical work. Diarylethene (DAE) derivative based nanowires were recently synthesized with template assisted slow solvent annealing and demonstrated to undergo mechanical bending with light exposure followed by the development of photomechanical fatigue as a result of multiple cycles of light exposures. ${ }^{1,2}$ This observed photomechanical fatigue hinders the usefulness of DAE nanowires in the applications of photomechanical actuators. This study investigates the origin of the photomechanical fatigue observed for DAE nanowires. Atomic Force Microscopy (AFM) nanoindentation technique was utilized to characterize the mechanical properties (Young's modulus) of DAE crystalline nanowires, macrodimensional crystals and amorphous films as a function of multiple cycles of light exposures. Our results indicate after approximately 20 light exposure cycles an individual DAE nanowire undergoes the crystalline to amorphous transformation. This transformation is evident from the powder X-ray diffraction data and measured Young's modulus values that display a remarkable four times decrease after the photomechanical fatigue of nanowires. Noteworthy, photomechanical fatigue is absent and thus sizedependent for a comparable number of light exposure cycles, likely due to a combination of different observed mechanical properties for macro- and nano-dimensional crystals and the cooperative nature of an extended crystal structure.

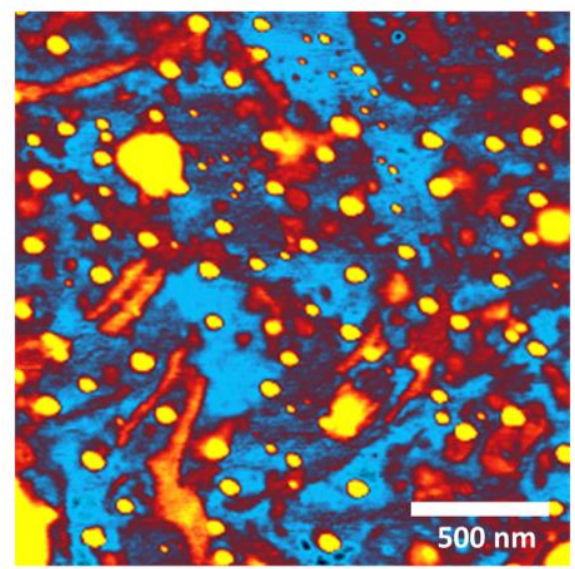

Figure 1. Atomic force microscopy height image of a diarylethene macro-dimensional crystal surface. 


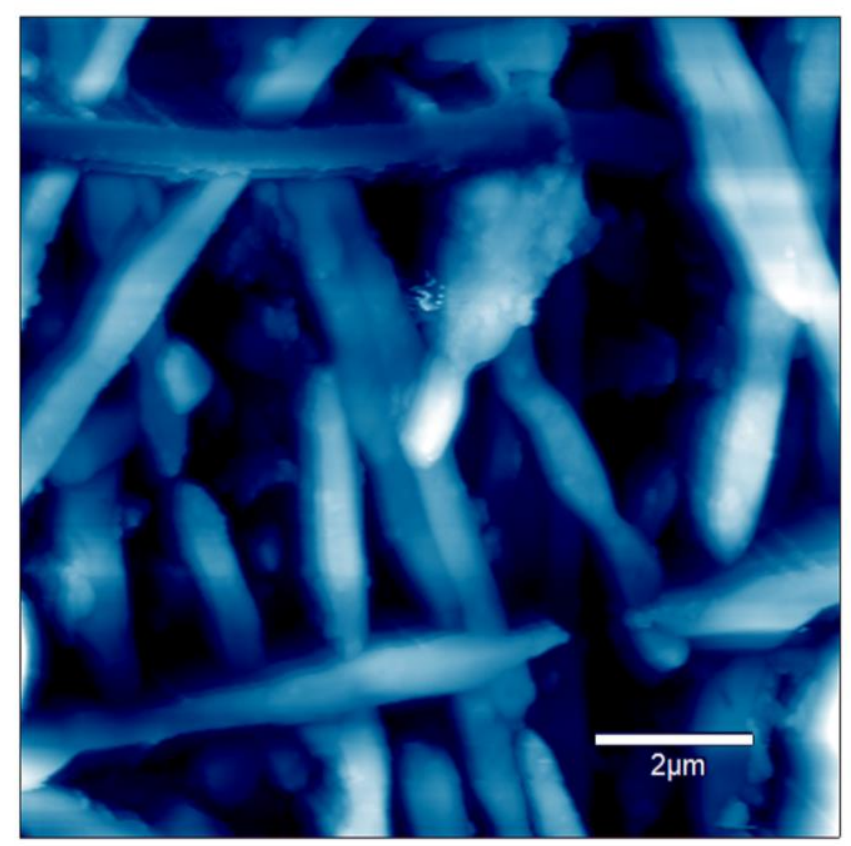

Figure 2. Atomic force microscopy height image of bundles of diarylethene nanowires deposited on an aluminum oxide template.

\section{References}

1. Tong, F.; Kitagawa, D.; Dong, X. N.; Kobatake, S.; Bardeen, C. J., Photomechanical motion of diarylethene molecular crystal nanowires. Nanoscale 2018, 10 (7), 3393-3398.

2. Dong, X. M.; Tong, F.; Hanson, K. M.; Al-Kaysi, R. O.; Kitagawa, D.; Kobatake, S.; Bardeen, C. J., Hybrid Organic Inorganic Photon-Powered Actuators Based on Aligned Diarylethene Nanocrystals. Chemistry of Materials 2019, 31 (3), 1016-1022. 